\title{
Rigorous feedback control of cAMP levels in Saccharomyces cerevisiae
}

\author{
Jun-ichi Nikawa, ${ }^{1}$ Scott Cameron, Takashi Toda, ${ }^{2}$ Kenneth $M$. Ferguson, and Michael Wigler
}

\begin{abstract}
We have isolated and characterized normal and mutant alleles of many of the genes of the RAS/adenylyl cyclase pathway of the yeast Saccharomyces cerevisiae. Manipulation of those genes has revealed a system for feedback control that can modulate cAMP levels over at least a 10,000-fold range. The feedback control depends upon the activity of the cAMP-dependent protein kinases and requires the presence of the CDC25 and RAS proteins. The capacity for such dramatic control of cAMP levels raises fundamental questions about the normal mechanism of action of the cAMP signaling system in yeast.
\end{abstract}

[Key Words: Saccharomyces cerevisiae; cAMP; RAS/adenylyl cyclase pathway; CDC25]

Fluctuations in cAMP concentrations play a major role in modulating the affairs of eukaryotic cells. In the yeast Saccharomyces cerevisiae, adenylyl cyclase activity is dependent upon the presence of either RAS1 or RAS2 proteins, and these proteins presumably modulate adenylyl cyclase (Broek et al. 1985; Toda et al. 1985). To investigate the interaction between adenylyl cyclase and $R A S$ proteins, we and others have cloned and mutated many of the genes of the RAS/adenylyl cyclase pathway, including $C D C 25$, the product of which probably modulates adenylyl cyclase through its effects on $R A S$ proteins (Broek et al. 1987; Robinson et al. 1987); RAS1 and RAS2, the two homologs of the mammalian ras oncogenes (DeFeo-Jones et al. 1983; Powers et al. 1984); CYR1, the gene encoding adenylyl cyclase (Matsumoto et al. 1982, 1984; Kataoka et al. 1985); TPK1, TPK2, and $T P K 3$, three genes that encode the catalytic subunits of the cAMP-dependent protein kinase (cAPK) (Toda et al. $1987 \mathrm{a}) ; B C Y 1$, the gene encoding the regulatory subunit of cAPK (Toda et al. 1987b); and PDE1 and PDE2, the genes encoding the low- and high-affinity cAMP phosphodiesterases, respectively (Londesborough and Suoranta 1983; Suoranta and Londesborough 1984; Sass et al. 1986; Nikawa et al. 1987). Measurements of cAMP levels in cells with disruptions of the PDE genes led us to suspect the existence of a negative feedback mechanism for controlling cAMP levels, because levels of cAMP were only two- to three-fold higher than wild type in strains lacking both phosphodiesterase genes (Nikawa et al. 1987). The experiments presented here demonstrate the existence of such a mechanism. Studies with mutant TPK and BCY1 genes indicate that the degree of feedback depends on the activity of cAPK. The modulation of cAMP levels achievable by this mechanism spans at least four orders of magnitude and is entirely dependent upon $R A S$ and $C D C 25$ proteins.
Results

Manipulation of $c A M P$ levels in phosphodiesterasedeficient strains.

We have measured cAMP levels in yeast strains deficient in cAMP phosphodiesterase (see Table 1 for strain descriptions). Levels were measured by radioimmunoassay in cultures grown to late exponential phase in rich medium. As we reported previously (Nikawa et al. 1987), cAMP levels are only slightly higher than wildtype levels in strains lacking both cAMP phosphodiesterase genes, PDE1 and PDE2 (see Table 2). Because PDE1 and PDE2 together encode the totality of detectable cAMP phosphodiesterase activity (J. Nikawa et al., in press), this suggests that either there is an additional pathway to rid cells of cAMP or there is a feedback mechanism that inhibits cAMP production in cells with even slightly elevated cAMP levels. Because we have shown that the $R A S$ proteins stimulate adenylyl cyclase activity (Broek et al. 1985; Toda et al. 1985), we compared PDE disruptions in strains with $R A S$ genes to strains lacking RAS genes (see Table 2). As we reported previously, pde $1^{-}$pde $2^{-}$ras $1^{-}$ras $2^{-}$cells are viable (Nikawa et al. 1987). Surprisingly, cAMP levels are actually somewhat higher in pde- strains DJ32-3B, DJ32-5B, and DJ32-8A, which lack both RAS genes, than in pdestrain DJ23-3C, which has both $R A S$ genes. This result is in sharp contrast to what we have observed for cells that possess intact $P D E$ genes. Such cells require $R A S$ proteins to maintain cAMP levels (Toda et al. 1985; see also Table 5). The result is explicable if $R A S$ proteins are not actively stimulating cAMP production in $\mathrm{pde}^{-}$ strains. This explanation favors a model whereby the increased cAMP in pde- strains feeds back to affect the machinery of cAMP production. 
Nikawa et al.

Table 1. Genotype of yeast strains used in this study

\begin{tabular}{|c|c|}
\hline Strain & Genotype \\
\hline $\mathrm{SP}^{\mathbf{a}}$ & MATa his3 leu2 ura3 trp1 ade8 can1 \\
\hline TK161-R2Vb & MATa his3 leu2 ura3 trp1 ade8 can1 RAS2val19 \\
\hline DJ23-3Cc & MAT $\alpha$ his3 leu2 ura3 trp1 ade8 pde1 : : LEU2 pde2 : : URA3 \\
\hline DJ31-4D & MAT $\alpha$ his3 leu2 ura3 trp1 ade8 RAS2 ${ }^{\mathrm{val19}}$ pde1 : : URA3 pde2 : : HIS3 \\
\hline DJ31-6Ad & MAT $\alpha$ his3 leu2 ura3 trp1 ade8 RAS2 ${ }^{\text {val19 }}$ pde1 : : URA3 pde2 : : HIS3 \\
\hline DJ32-3Be & MATa his3 leu2 ura3 trp1 ade8 ras1 : : HIS3 ras2 : : ADE8 pde1 : LEU2 pde2 : : URA3 \\
\hline DJ32-5Be & 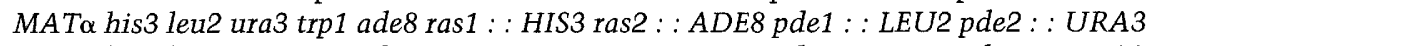 \\
\hline DJ32-8A & MATa his3 leu2 ura3 trp1 ade 8 ras $1::$ HIS3 ras $2::$ ADE8 pde1 $::$ LEU2 pde2 : : URA3 \\
\hline$S 7-1 A^{f}$ & MAT $\alpha$ his3 leu2 ura3 trp1 ade8 tpk2 : : HIS3 tpk3 : : TRP1 \\
\hline S7-1B & MAT $\alpha$ his3 leu2 ura3 trp1 ade8 tpk1 : : URA3 tpk3 : : TRP1 \\
\hline S7-7A & MATa his3 leu2 ura3 trp1 ade8 tpk2 : : HIS3 tpk3 : : TRP1 \\
\hline S7-3C & MATa his3 leu2 ura3 trp1 ade8 tpk1 : : URA3 tpk2 : : HIS3 \\
\hline S13-3A & MAT $\alpha$ his3 leu2 ura3 trp1 ade8 tpk2 : : HIS3 tpk3 : :TRP1 bcy1 : : LEU2 \\
\hline $\mathrm{S} 13-7 \mathrm{C}$ & MATa his3 leu2 ura3 trp1 ade8 tpk1 : : URA3 tpk3 : : TRP1 bcy1 : : LEU2 \\
\hline S13-58A & MATa his3 leu2 ura3 trp1 ade8 tpk2 : : HIS3 tpk3 : : TRP1 bcy1 : : LEU2 \\
\hline S15-1D & MATa his3 leu2 ura3 trp1 ade8 tpk1 : URA3 tpk2 ${ }^{\mathrm{w} 1}$ tpk3 $::$ TRP1 bcy1 : : LEU2 \\
\hline S15-5B & MATa his3 leu2 ura3 trp1 ade8 tpk1 : : URA3 tpk2 ${ }^{\text {w1 }}$ tpk3 : : TRP1 \\
\hline S18-1D & MAT $\alpha$ his3 leu2 ura3 trp1 ade8 tpk1 ${ }^{\text {wl }}$ tpk2 $::$ HIS3 tpk3 : : TRP1 \\
\hline $\mathrm{S} 18-3 \mathrm{~B}$ & MATa his3 leu2 ura3 trp1 ade8 tpk1 ${ }^{\text {w1 }}$ tpk2 : : HIS3 tpk3 : : TRP1 \\
\hline S22-5D & MATa his3 leu2 ura3 trp1 ade8 tpk1 : : URA3 tpk2 : : HIS3 tpk $3^{\mathrm{wl}}$ \\
\hline S22-9A & MATa his3 leu2 ura3 trp1 ade8 tpk1 : URA3 tpk2 : : HIS3 tpk3 ${ }^{\mathrm{w} 1}$ bcy1 $::$ LEU2 \\
\hline RS13-7C-1 & MATa his3 leu2 ura3 trp1 ade8 tpk1 : : URA3 tpk2 ${ }^{\text {w1 }}::$ tpk3 TRP1 bcy1 : : LEU2 \\
\hline RS13-58A-1 & MATa his3 leu2 ura3 trp1 ade8 tpk1 ${ }^{\text {w1 }}$ tpk2 : : HIS3 tpk3 : : TRP1 bcy1 : : LEU2 \\
\hline TF3.1 & MATa his3 leu2 ura3 trp1 ade8 tpk1 : : URA3 tpk2 : : HIS3 bcy1 : :LEU2 \\
\hline RTF3.1-1 & MATa his3 leu2 ura3 trp1 ade8 tpk1 : : URA3 tpk2 : : HIS3 tpk3 w1 bcy1 : : LEU2 \\
\hline TF16.1 & MATa his3 leu2 ura3 trp1 ade8 tpk1 ${ }^{\text {w1 }}$ tpk2 : : HIS3 tpk3 : : TRP1 bcy1 : : LEU2 cdc25 : : URA3 \\
\hline TF18.2 & MATa his3 leu2 ura3 trp1 ade8 tpk1w1 tpk2 : : HIS3 tpk3 : : TRP1 bcy1 : LEU2 ras $1::$ URA3 ras2 : : ADE8 \\
\hline RTF1.5-1 & MATa his3 leu2 ura3 trp1 ade8 tpk1 ${ }^{\text {w2 }}$ tpk2 : : HIS3 tpk3 : : TRP1 bcy1 : : URA3 \\
\hline
\end{tabular}

${ }^{\text {a }}$ From Cold Spring Harbor Laboratory collection.

b TK161-R2V was described previously (Toda et al. 1985).

${ }^{c} \mathrm{DJ} 23-3 \mathrm{C}$ was described previously (J. Nikawa et al., in press).

dDJ31-4D and -6A are segregants of diploid DJ31 (MATa/MATa leu2/leu2 his3/his3 ura3/ura3 trp1/trp1 ade8/ade8 RAS2val19/ ras2 : : ADE8 PDE1/pde1 : : URA3 PDE2/pde2 : : HIS3). Because diploid strains containing RAS2 $2^{\text {valls }}$ fail to sporulate, we first transformed DJ31 with a high-copy plasmid containing LEU2 and the gene JUN1. JUN1 is a suppressor of RAS2 ${ }^{\text {val19 }}$ (J. Nikawa, unpubl.) that restores sporulation to such diploids. Haploid progeny were thus obtained and, subsequently, segregants were picked that had lost the LEU2 JUN1 genes.

${ }^{e}$ DJ32-3B, -5B, and -8A are segregants of a diploid DJ32 (J. Nikawa et al., in press).

${ }^{f}$ Constructions of this and following strains will be described elsewhere (S. Cameron et al., in prep:). Strains beginning with $S$ denote segregants from diploids; those beginning with TF denote strains picked after transformation with DNA; those beginning with $\mathrm{R}$ denote strains derived by mutant selection. The superscripts w1 or w 2 denote attentuated alleles of the various $T P K$ genes.

To search further for evidence of feedback inhibition, we examined the effects of the mutant $R A S 2^{\text {val19 }}$ gene in $p d e^{-}$strains. The $R A S 2^{\text {vall }}$ mutation, as described previously (Kataoka et al. 1984), encodes a RAS2 protein with valine substituted for glycine at position 19 . This substitution is analogous to one found in oncogenic mammalian ras proteins. Cells containing $R A S 2^{\text {valls }}$ show an abnormal response to nutrient stresses: They fail to sporulate, to arrest properly in $G_{1}$, to survive prolonged starvation, to accumulate glycogen, and to become heat shock resistant (Kataoka et al. 1984; Toda et al. 1985). When cAMP phosphodiesterases are present, cAMP levels are only a few-fold higher in $R A S 2^{\text {vall } 9}$ strains than in RAS2 strains. However, strains that are $R A S 2^{\text {val19 }}$ pde1- $^{-}$pde2- have 1000-fold higher cAMP levels than strains that are $p d e 1^{-}$pde2- but contain wild-type RAS2 (see Table 3). Under these conditions, therefore, adenylyl cyclase must be considerably more active in the presence of $R A S 2^{\text {vallo }}$ protein than it is in the presence of wild-type $R A S 2$ protein. The level of
cAMP in the $R A S 2^{\text {valli }} p d e 1^{-} p d e 2^{-}$strains is quite remarkable. If we estimate that 1 gram of yeast contains $100 \mathrm{mg}$ of protein, the intracellular cAMP concentration in $R A S 2^{\text {vallo }}$ pde $1^{-}$pde $2^{-}$strains could be as high as 0.35 $\mathrm{mM}$, or perhaps $20 \%$ of the concentration of intracellular ATP. This result provides conclusive evidence of the existence of a feedback mechanism for controlling cAMP levels and suggests that $R A S 2^{\text {val19 }}$ protein can at least partially override feedback inhibition.

\section{Manipulating $c A M P$ levels by altering the cAMP.} dependent protein kinases

The above experiments indicate the existence of a powerful feedback system for the control of cAMP levels. To test the idea that feedback might be linked to the activity of the CAPK, rather than to CAMP levels per se, we measured levels of cAMP in cells containing mutations in the genes encoding the components of the cAPK. In yeast, there appears to be a single gene, $B C Y 1$, that en- 
Table 2. Intracellular cAMP levels of RAS and PDE mutants

\begin{tabular}{|c|c|c|c|c|c|}
\hline \multirow[b]{2}{*}{ Strain ${ }^{a}$} & \multicolumn{4}{|c|}{ Genotype $^{a}$} & \multirow{2}{*}{$\begin{array}{l}\text { cAMP level } \\
\text { (pmole/mg protein }\end{array}$} \\
\hline & $R A S 1$ & RAS2 & PDE1 & PDE2 & \\
\hline SP1 & + & + & + & + & 1.7 \\
\hline DJ23-3C & + & + & - & - & 2.7 \\
\hline DJ32-3B & - & - & - & - & 5.9 \\
\hline DI32-5B & - & - & - & - & 5.4 \\
\hline DJ32-8A & - & - & - & - & 5.4 \\
\hline
\end{tabular}

a Complete genotypes of the yeast strains are given in Table 1.

b Cells were cultured in rich medium (YPD) and used for the cAMP determination, as described in Experimental procedures.

codes the regulatory subunit of the cAPKs (Toda et al. 1987b). Three genes, TPK1, TPK2, and TPK3, encode the catalytic subunits (Toda et al. 1987). Complete disruption of the $B C Y 1$ gene leads to a severe phenotype characterized by failure to grow on nonglucose carbon sources, failure to sporulate, failure to survive nutrient starvation, failure to accumulate glycogen, failure to arrest in $\mathrm{G}_{1}$, and failure to become heat shock resistant in stationary phase (Toda et al. 1987b). Other mutant $B C Y 1$ alleles exist with less severe phenotypes (Matsumoto et al. 1982). The effects of complete disruption of the $B C Y 1$ gene are probably due to the resulting unbridled activity of the CAPK catalytic subunits because mutations within the TPK genes completely abolish these bcy1effects (S. Cameron., in prep.). Such intragenic TPK mutations were obtained by us in the following way. First, we created strains that contained only a single TPK gene (e.g., TPK1 tpk2- $t p k 3^{-}$) and a disrupted BCY1 gene. These cells have the $b c y 1^{-}$phenotype. From these cells we obtained mutants that had suppressed the bcy $1^{-}$ phenotype. All such cells contained single recessive mutations in the remaining viable TPK gene. The proteins produced by these mutant alleles of $T P K$ genes display greatly reduced catalytic activity in vitro. The mutant $T P K$ genes we thus obtained are therefore attenuated and we denote them $t p k 1^{\mathrm{w}}, t p k 2^{\mathrm{w}}$, and $t p k 3^{\mathrm{w}}$. A complete description of this work is given elsewhere (S. Cameron, in prep.).

As before, we measured cAMP levels in various strains containing mutations in the genes encoding components of the cAPK (Table 4). Strains with a completely disrupted $B C Y 1$ have a pronounced decrease in cAMP levels, provided that such strains have at least one intact TPK gene. We have not previously seen such a drop in cAMP levels in strains carrying a mutant (but not disrupted) $b c y 1$ allele, or in cells containing the TPK genes on multicopy plasmids. We attribute this new observation to the severity of the phenotype resulting from the complete disruption of $B C Y 1$ and take it as evidence that greatly enhanced activity of the TPK proteins lowers cAMP levels. Conversely, strains that do not express wild-type TPK-gene products, such as S18-3B

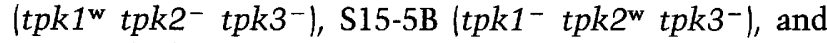
S22-5D $\left(t p k 1^{-} t p k 2^{-} t p k 3^{w}\right)$, have elevated cAMP levels dramatically, compared with wild-type cells. The absence of the BCY1 gene in $t p k^{w}$ strains changes the picture little, consistent with the notion that the altered cAPK catalytic subunits of $t p k^{w}$ have weak activity even in the absence of the regulatory subunit. In the case of S18-3B and S15-5B, the cAMP levels are elevated about 1000-fold compared with wild-type strains. We attribute this increase to the near absence of feedback inhibition resulting from a lack of any fully active cAPK catalytic subunits. Thus, the full excursion of cAMP levels observable by manipulating the cAPK system is at least 10,000 -fold. The concentration of cAMP in the tpkw strains is quite remarkable, especially considering that the $P D E$ genes are intact. In fact, the cAMP concentration in these $t p k^{w}$ strains is in the vicinity of the reported $K_{\mathrm{m}}$ value of the low-affinity cAMP phosphodiesterase (Londesborough and Lukkari et al. 1980).

Remarkably, the elevation of cAMP levels observable in a $t p k 1^{w}$ strain is entirely dependent on both $R A S$ and $C D C 25$ genes (Table 5). Neither RAS nor CDC25, nor-

Table 3. Intracellular cAMP Levels of RAS and PDE mutants

\begin{tabular}{|c|c|c|c|c|c|c|c|}
\hline \multirow[b]{2}{*}{ Strain $^{a}$} & \multicolumn{4}{|c|}{ Genotype $^{\mathbf{a}}$} & \multicolumn{3}{|c|}{$\begin{array}{c}\text { cAMP level }{ }^{\mathrm{b}} \\
\text { (pmole/mg protein) }\end{array}$} \\
\hline & $R A S 1$ & RAS2 & PDE1 & PDE2 & exp. 1 & exp. 2 & exp. 3 \\
\hline SP1 & + & G & + & + & 1.7 & 1.6 & 1.8 \\
\hline TK161-R2V & + & $\mathrm{V}$ & + & + & 2.7 & ND & 3.5 \\
\hline DJ23-3C & + & G & - & - & 3.6 & ND & ND \\
\hline DJ31-4D & + & $\mathrm{V}$ & - & - & 2300 & 450 & 3000 \\
\hline DJ31-6A & + & $\mathrm{V}$ & - & - & ND & 3500 & ND \\
\hline
\end{tabular}

a Complete genotypes of the yeast strains are given in Table 1. (G) The wild-type allele, with glycine at amino acid position 19; (V) the $R A S 2^{\text {val19 }}$ allele, with valine at amino acid position 19 .

b Cells were cultured in rich medium (YPD) and used for the cAMP determination, as described in Experimental procedures. (ND) Not determined. 
Nikawa et al.

Table 4. Effects of mutant TPK and BCY1 genes on cAMP levels

\begin{tabular}{|c|c|c|c|c|c|}
\hline \multirow[b]{2}{*}{ Strain $^{a}$} & \multicolumn{4}{|c|}{ Genotype $^{a}$} & \multirow{2}{*}{$\begin{array}{l}\text { cAMP levelb } \\
\text { (pmole/mg protein) }\end{array}$} \\
\hline & $T P K 1$ & TPK2 & TPK3 & $B C Y 1$ & \\
\hline S7-1A & + & - & - & + & 2.5 \\
\hline S7-7A & + & - & - & + & 1.8 \\
\hline S13-58A & + & - & - & - & 0.4 \\
\hline S13-3A & + & - & - & - & 0.5 \\
\hline S18-3B & wl & - & - & + & 1500 \\
\hline S18-1D & w1 & - & - & + & 1800 \\
\hline RS13-58A-1 & wl & - & - & - & 570 \\
\hline RTF1.5-1 & w2 & - & - & - & 120 \\
\hline S7-1B & - & + & - & + & 3.7 \\
\hline S13-7C & - & + & - & - & 0.3 \\
\hline S15-5B & - & wl & - & + & 3400 \\
\hline RS13-7C-1 & - & wl & - & - & 3400 \\
\hline S15-1D & - & wl & - & - & 3800 \\
\hline$S 7-3 C$ & - & - & + & + & 4.3 \\
\hline TF3.1 & - & - & + & - & 0.2 \\
\hline S22-5D & - & - & wl & + & 110 \\
\hline RTF3.1-1 & - & - & wl & - & 110 \\
\hline S22-9A & - & - & wl & - & 66 \\
\hline
\end{tabular}

a Complete genotypes of the yeast strains are given in Table 1. The mutant attenuated tpk ${ }^{\mathrm{w}}$ alleles described in the text.

b Cells were cultured in rich medium (YPD) and used for the cAMP determination, as described in Experimental procedures.

mally essential genes (Kataoka et al. 1984; Tatchell et al. 1984; Broek et al. 1987; Robinson et al. 1987), are required for the viability of $b c y 1^{-} t p k 1^{\mathrm{w}} t p k 2^{-} t p k 3^{-}$ strains (S.Cameron, in prep.). Levels of cAMP in TF18.2

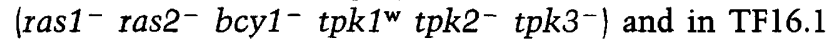
(cdc25- bcy1- tpk1w tpk2- tpk3-) are below our limit of detection. This result indicates an extraordinarily rigorous control of adenylyl cyclase activity by RAS and $C D C 25$ proteins. Moreover, if the CDC25 product controls the activity of $R A S$ proteins (Broek et al. 1987; Robinson et al. 1987), this control is just as rigorous.

The elevation in cAMP levels seen in strains carrying attenuated TPK proteins could be explained by an effect on cAMP production, or on the CAMP phosphodiesterases, or both. To test for effects on the cAMP phosphodiesterases, we measured levels of activity of both the low- and the high-affinity cAMP phosphodiesterases in the low cAMP strain S13-58A (bcy1- TPK1 tpk2tpk3-) and in the high cAMP strain RS13-58A-1 (bcy $1^{-}$ tpk $1^{\mathrm{w}}$ tpk2- $t p k 3^{-}$) (Table 6). Phosphodiesterase activities were nearly identical in both strains.

\section{Discussion}

We have observed that the levels of cAMP in a growing population of cells can be modulated over at least a 10,000 -fold range, simply by genetically manipulating the activity of the cAMP-dependent protein kinases. Low levels of cAMP are found in bcy $1^{-}$strains containing wild-type catalytic subunits of cAPK, and enormously elevated levels of CAMP are found in strains containing only attenuated CAPK catalytic subunits $\left(t p k^{w}\right)$. Thus, it is highly likely that cAMP levels in cells are controlled by a powerful feedback mechanism that is dependent upon the activity of cAPK. It is clearly possible that CAPK itself feeds back directly on components of the cAMP generating system. There is ample precedent for feedback control of components of signaling systems by protein phosphorylation (Sibley et al. 1987). Both the $C D C 25$ and adenylyl cyclase proteins contain multiple consensus sites for cAPK phosphorylation in their amino-terminal domains (Kataoka et al. 1985; Camonis et al. 1986; Broek et al. 1987).

Feedback control can, in principle, affect the rate of

Table 5. Requirement of RAS and CDC25 for the production of $c A M P$ in tpk ${ }^{\mathrm{w}}$ strains

\begin{tabular}{lccccccccc}
\hline & \multicolumn{7}{c}{ Genotype $^{\mathrm{a}}$} & cAMP level \\
\cline { 2 - 8 } Strain $^{\mathrm{b}}$ & TPK1 & TPK2 & TPK3 & BCY1 & RAS1 & RAS2 & CDC25 & (pmole/mg protein) \\
\hline TF16.1 & w1 & - & - & - & + & + & - & 0.2 \\
TF18.2 & wl & - & - & - & - & - & + & +3 \\
RS13-58A-1 & w1 & - & - & - & + & + & + & 570 \\
\hline
\end{tabular}

a See footnote a in Table 4.

b See footnote $b$ in Table 4. 
Table 6. $\quad$ AMP phosphodiesterase activities in TPK1 BCY1 mutant strains

\begin{tabular}{|c|c|c|c|c|}
\hline \multirow[b]{2}{*}{ Strain $^{a}$} & \multicolumn{2}{|c|}{ Genotype $^{a}$} & \multicolumn{2}{|c|}{$\begin{array}{l}\text { Phosphodiesterase activity } \\
\text { (nmole/min/mg protein) }\end{array}$} \\
\hline & TPK1 & $B C Y 1$ & low-affinity & high-affinity \\
\hline S7-1A & + & + & 1.0 & 0.065 \\
\hline S18-1D & wl & + & 1.2 & 0.060 \\
\hline S13-58A & + & - & 1.1 & 0.058 \\
\hline RS13-58A-1 & wl & - & 1.0 & 0.043 \\
\hline
\end{tabular}

a See footnote a in Table 4.

b Cells were cultured in rich medium (YPD) and used for the enzyme activity determination, as described in Experimental procedures. Low- and high-affinity phosphodiesterases are the gene products of $P D E 1$ and PDE2, respectively.

generation of cAMP, its rate of loss, or both. Two lines of evidence indicate that feedback occurs at least at the level of cAMP generation. First, attenuation of the TPK genes produces a far greater increase in cAMP levels than does the elimination of the cAMP phosphodiesterase genes. Thus, adenylyl cyclase activity in $t p k^{\mathrm{w}}$ strains must exceed the activity in $p d e^{-}$strains. Second, unlike wild-type strains, maintenance of cAMP levels in $p d e^{-}$strains does not require the presence of "RAS proteins. This suggests that the activity of RAS proteins in such strains is already at a minimum due to feedback inhibition and that the cAMP found in $p d e^{-}$cells results from an unstimulated, basal activity of adenylyl cyclase. In contrast to cAMP production, we cannot infer that feedback affects the rate of loss of cAMP. We observed no difference in the cAMP phosphodiesterase activity between strains with high and low cAPK activity (Table 6). Unlike the $C D C 25$ and adenylyl cyclase proteins, we find no consensus sites for CAPK phosphorylation in either the PDE1 or PDE2 proteins (Sass et al. 1986; Nikawa et al. 1987a). If, however, the activity of the cAMP phosphodiesterases is not modulated by feedback control, we are left with several uncomfortable conclusions about the capacity of the cAMP generating system, among them that, at its maximum, the cell can generate so much cAMP that the capacity of the CAMP phosphodiesterases is overwhelmed. Obviously, considerably more work is required before we can understand the pathways of feedback control.

Our results highlight some remarkable features of the functions of the RAS proteins. First, as mentioned above, it is likely that the normal RAS protein, under certain physiological conditions, is subject to feedback control. Under the same conditions wherein the wildtype RAS2 protein appears to be down-regulated, the $R A S 2^{\text {vall9 }}$ protein is highly active (Table 2 ). This suggests that the $R A S 2^{\text {val19 }}$ protein may be able to override feedback control. In fact, experiments with cultured animal cells microinjected with normal and oncogenic H-ras proteins also suggest that there is a feedback mechanism for controlling the activity of normal $\mathrm{H}$-ras protein that fails to control the oncogenic protein (Bar-Sagi and Feramisco 1986). Second, although it is possible to raise cAMP levels by 1000 -fold in mutant $t p k^{\mathrm{w}}$ strains, cAMP levels are below our limit of detection in mutant $t p k^{w}$ strains that lack $R A S$ proteins. Thus, the cAMP levels in these two types of strains differ by four orders of magnitude. RAS proteins must therefore exert an astonishingly stringent control over adenylyl cyclase. Third, the $C D C 25$ product exhibits as tight a control over cAMP levels as do the RAS proteins. If, as seems likely, the CDC25 product controls RAS proteins (Broek et al. 1987; Robinson et al. 1987), this control must also be extraordinarily stringent. We are left with the impression of an almost absolute chain of command from the $C D C 25$ product to the RAS proteins to adenylyl cyclase.

We have demonstrated that yeast cells can adjust their cAMP levels in response to chronic changes in their cAMP signaling pathway. This observation may explain why yeast cells are relatively resistant to mutations affecting components of the cAMP signaling pathway. For example, yeast can lose any two of their three cAPK catalytic subunits without an apparent change in phenotype (Toda et al. 1987a). Cells may compensate for this loss in cAPK catalytic subunits by increasing cAMP levels (Table 4). Again, disruption of either phosphodiesterase gene results in a very minimal phenotype and only a small change in cAMP levels (Sass et al. 1986; J. Nikawa et al., in press). In principle, this cAMP feedback system could render yeast cells able to sense changes in the levels of a stimulus against a chronic background of stimulation. Just such a mechanism allows for light adaptation in the visual transduction system (Sibley et al. 1987). We should emphasize, however, that we have not demonstrated feedback for acute changes. Indeed, we have no knowledge from our experiments about the dynamic parameters of the feedback response. Nevertheless, it is clearly possible that feedback could rapidly dampen acute responses to stimuli, and this may explain the rapid biphasic changes in cAMP level seen in starved cells in response to glucose feeding (Purwin et al. 1982; Thevelein and Beullens 1985).

\section{Experimental procedures}

Strains, media, yeast genetics, and yeast genetic nomenclature

S. cerevisiae strains used are listed in Table 1. Escherichia coli $\mathrm{HB} 101$ was used for plasmid propagation and isolation. Yeast cells were grown in YPD (rich) media ( $1 \%$ Bacto-yeast extract, $2 \%$ Bacto-peptone, and $2 \%$ dextrose/ at $30^{\circ} \mathrm{C}$. General genetic manipulation of yeast cells was carried out as described (Mortimer and Hawthorne 1969). Wild-type alleles are denoted by 
capital italicized letters, dominant mutations are denoted by capital italicized letters, recessive mutations by lowercase italicized letters, and gene disruptions by lowercase letters representing the disrupted gene followed by two colons and the wild-type gene marker. For example ras1 : : HIS3 indicates the RAS1 gene disrupted by the HIS3-gene marker. In the text of this paper, gene disruptions are abbreviated by lowercase italicized letters representing the gene followed by a superscript minus sign, such as ras ${ }^{-}$.

\section{Construction of mutants}

Construction of the mutant alleles of TPK genes will be described in detail elsewhere (Cameron et al., in prep.).

\section{DNA and plasmid constructions}

Plasmid DNA was isolated from E. coli by the alkali lysis method (Maniatis et al. 1982). DNA restriction endonucleases, polymerases, and ligases were used under conditions recommended by suppliers (New England Biolabs or Bethesda Research Labs). ppde1 : : URA3 was constructed by the same method used for ppde1 : : LEU2 (Nikawa et al. 1987), except the 1.1-kb HindIII fragment of $U R A 3$ gene was used instead of the LEU2 gene. ppde2 : : HIS3 was constructed by the same method used for ppde2 : : URA3 (Sass et al. 1986), except the $1.7-\mathrm{kb} B a m H I$ fragment of the HIS3 gene was used instead of the URA3 gene. For gene-disruption experiments, suitable linear DNA fragments were isolated and used for transformation of yeast cells, as described (Rothstein 1983).

\section{cAMP determination}

Yeast strains were grown in YPD medium to a cell density of approximately $10^{8}$ cells $/ \mathrm{ml}$. Cells were harvested by centrifugation and resuspended in $1 \mathrm{ml} 5 \%$ trichloroacetic acid. Cells were disrupted by vortexing in the presence of glass beads. The homogenized sample was centrifuged to remove the insoluble materials, and the supernatant was extracted with ether and lyophilized. The lyophylized sample was dissolved in water, and the cAMP content was determinated by radioimmunoassay (Harper and Brooker 1975). Protein concentration of whole cells was determined by the method of Lowry et al. (Lowry et al. 1951) after cells were treated with $1 \mathrm{M} \mathrm{NaOH}$.

\section{Determinations of phosphodiesterase activities}

Yeast cells were cultured at $30^{\circ} \mathrm{C}$ in YPD medium. Cell homogenates were prepared as described previously (Sass et al. 1986) and used for the determination of phosphodiesterase activities. Low- and high-affinity phosphodiesterase activities were assayed as described (Sass et al. 1986), using $0.5 \mathrm{mM}$ cAMP and 1 $\mu \mathrm{M}$ cAMP as substrate, respectively.

\section{Acknowledgments}

We thank Drs. J. Harper and G. Brooker for their generous assistance. We also thank P. Bird for her help in preparation of this manuscript. S.C. is a graduate student in the Genetics Program at SUNY Stony Brook. This work was supported by the National Institutes of Health, the American Business Foundation for Cancer Research, the American Cancer Society, and the Pfizer Biomedical Research Award. M.W. is an American Cancer Society research professor.

\section{References}

Bar-Sagi, D. and J.R. Feramisco. 1986. Induction of membrane ruffling and fluid-phase pinocytosis in quiescent fibroblasts by ras proteins. Science 233: $1061-1068$.

Broek, D., N. Samiy, O. Fasano, A. Fujiyama, F. Tamanoi, J. Northup, and M. Wigler. 1985. Differential activation of yeast adenylate cyclase by wild-type and mutant ras proteins. Cell 41: 763-769.

Broek, D., T. Toda, T. Michaeli, L. Levin, C. Birchmeier, M. Zoller, S. Powers, and M. Wigler. 1987. The S. cerevisiae $C D C 25$ gene product regulates the RAS/adenylate cyclase pathway. Cell 48: 789-799.

Camonis, J.H., M. Kalekine, G. Bernard, H. Garreau, E. BoyMarcotte, and M. Jacquet. 1986. Characterization, cloning, and sequence analysis of the $C D C 25$ gene which controls the cyclic AMP level of Saccharomyces cerevisiae. EMBO I. 5: 375-380.

DeFeo-Jones, D., E.M. Scolnick, R. Koller, and R. Dhar. 1983. ras-related gene sequences identified and isolated from Saccharomyces cerevisiae. Nature. 306: 707-709.

Harper, J.F. and G.J. Brooker. 1975. Femtomole sensitive radioimmunoassay for cAMP and cGMP after 2'O acylation by acetic anhydride in aqueous solution. $J$. Cyclic Nucleotide Research 1: 207-218.

Kataoka, T., D. Broek, and M. Wigler. 1985. DNA sequence and characterization of the $S$. cerevisiae gene encoding adenylate cyclase. Cell 43: 493-505.

Kataoka, T., S. Powers, C. McGill, O. Fasano, J. Strathern, J. Broach, and M. Wigler. 1984. Genetic analysis of yeast RAS1 and RAS2 genes. Cell 37: 437-445.

Londesborough, J. and T-M. Lukkari. 1980. The $\mathrm{pH}$ and temperature dependence of the activity of the high $\mathrm{Km}$ cyclic nucleotide phosphodiesterase of bakers yeast. $I$. Biol. Chem. 255: 9262-9267.

Londesborough, J. and K. Suoranta. 1983. The zinc-containing high $\mathrm{K}_{\mathrm{m}}$ cyclic nucleotide phosphodiesterase of bakers' yeast. I. Biol. Chem. 258: 2966-2972.

Lowry, O., N. Rosebrough, A. Farr, and R. Randall. 1951. Protein measurement with a phenol reagent. $I$. Biol. Chem. 193: $265-275$.

Maniatis, T., E.F. Fritsch, and J. Sambrook. 1982. In: Molecular cloning: A laboratory manual. Cold Spring Harbor Laboratory, Cold Spring Harbor, New York.

Matsumoto, K., I. Uno, and T. Ishikawa. 1984. Identification of the structural gene and nonsense allele for adenylate cyclase in Saccharomyces cerevisiae. J. Bacteriol. 157: 277-282.

Matsumoto, K., I. Uno, A. Toh-e, T. Ishikawa, and Y. Oshima. 1982. Cyclic AMP may not be involved in catabolite repression in Saccharomyces cerevisiae: Evidence from mutants capable of using it as an adenine source. J. Bacteriol. 150: $277-285$.

Mortimer, R.K. and D.C. Hawthorne. 1969. The yeast (ed. A.H. Rose and J.S. Harrison). vol. 1, pp. 385-460. Academic Press, New York.

Nikawa, J., P. Sass, and M. Wigler. 1987. The cloning and characterization of the low affinity cAMP phosphodiesterase gene of $S$. cerevisiae. Molecular and Cellular Biology, 7: 3629-3636.

Powers, S., T. Kataoka, O. Fasano, M. Goldfarb, J. Strathern, J. Broach, and M. Wigler. 1984. Genes in S. cerevisiae encoding proteins with domains homologous to the mammalian ras proteins. Cell 36: 607-612.

Purwin, C., F. Leidig and H. Holzer. 1982. Cyclic AMP-dependent phosphorylation of fructose-1,6-bisphosphatase in yeast. Biochem. Biophys. Res. Comm. 107: 1482-1489.

Robinson, L., J. Gibbs, M. Marshall, I. Sigal, and K. Tatchell. 
1987. CDC25: A component of the RAS-adenylate cyclase pathway in Saccharomyces cerevisiae. Science. 255: 12181221.

Rothstein, R. 1983. One step gene disruption in yeast. Methods Enzymol. 101: 202-211.

Sass, P., J. Field, J. Nikawa, T. Toda, and M. Wigler. 1986. Cloning and characterization of the high affinity cAMP phosphodiesterase of S. cerevisiae. Proc. Natl. Acad. Sci. 83: 9303-9307.

Sibley, D., J. Benovic, M. Caron, and R. Lefkowitz. 1987. Regulation of transmembrane signaling by receptor phosphorylation. Cell 48: 913-922.

Suoranta, K. and J. Londesborough. 1984. Purification of intact and nicked forms of a zinc containing $\mathrm{Mg}^{2+}$-dependent, low $K_{\mathrm{m}}$ cyclic AMP phosphodiesterase from bakers' yeast. $J$. Biol. Chem. 259: 6964-6971.

Tatchell, K., D. Chaleff, D. Defeo-Jones, and E. Scolnick. 1984. Requirement of either of a pair of ras related genes of Saccharomyces cerevisiae for spore viability. Nature 309: 523527.

Thevelein, J. and M. Beullens. 1985. Cyclic AMP and the stimulation of trehalose activity in the yeast Saccharomyces cerevisiae by carbon sources, nitrogen sources, and inhibitors of protein synthesis. J. Gen. Microbiol. 131: 3199-3209.

Toda, T., S. Cameron, P. Sass, and M. Wigler. 1987a. Three different genes in the yeast Saccharomyces cerevisiae encode the catalytic subunits of the cAMP dependent protein kinase. Cell 50: 277-287.

Toda, T., S. Cameron, P. Sass, M. Zoller, J.D. Scott, B. McMullen, M. Hurwitz, E.G. Krebs, and M. Wigler. 1987b. Cloning and characterization of $B C Y 1$, a locus encoding a regulatory subunit of the cAMP-dependent protein kinase in yeast. Mol. Cell. Biol. 7: 1371-1377.

Toda, T., I. Uno, T. Ishikawa, S. Powers, T. Kataoka, D. Broek, S. Cameron, J. Broach, K. Matsumoto, and M. Wigler. 1985. In yeast, $R A S$ proteins are controlling elements of adenylate cyclase. Cell 40: 27-36. 


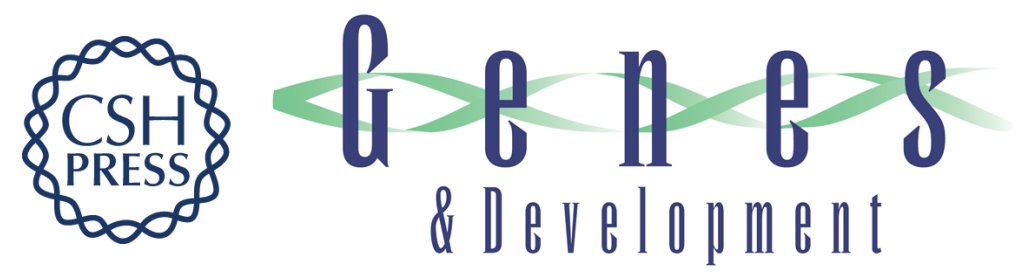

\section{Rigorous feedback control of cAMP levels in Saccharomyces cerevisiae.}

J Nikawa, S Cameron, T Toda, et al.

Genes Dev. 1987, 1:

Access the most recent version at doi:10.1101/gad.1.9.931

References This article cites 26 articles, 10 of which can be accessed free at: http://genesdev.cshlp.org/content/1/9/931.full.html\#ref-list-1

\section{License}

Email Alerting

Receive free email alerts when new articles cite this article - sign up in the box at the top Service right corner of the article or click here.

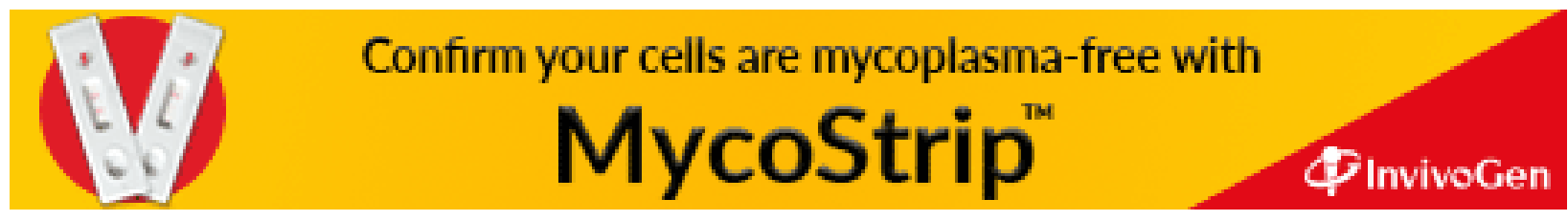

